\title{
Ein Extremalproblem für einbeschriebene Dreiecke
}

\author{
Christian Schütz \\ Christian Schütz studierte Mathematik und Geographie an den Universitäten in \\ Bochum und Köln. Seit 1978 ist er im Schuldienst in Nordrhein-Westfalen tätig. \\ Gegenwärtig arbeitet er als Studiendirektor mit den Fächern Mathematik, Informatik \\ und Erdkunde an einem Gymnasium in Krefeld.
}

Die vorliegende Arbeit wurde durch eine elementargeometrische Vermutung von N.D. Kazarinoff aus dem Jahre 1961 angeregt. Diese Vermutung wird hier in einen erweiterten Problemzusammenhang gestellt, und das Kazarinoff-Problem wird gemeinsam mit verwandten Problemen gelöst.

Es sei $\Delta=A B C$ ein Dreieck, dem ein anderes Dreieck $\Delta_{E}=X Y Z$ derart einbeschrieben ist, dass auf jeder Seite von $A B C$ genau eine Ecke von $X Y Z$ liegt. Wir nennen $X Y Z$ dann ein einbeschriebenes Dreieck oder kürzer ein Innendreieck $\Delta_{E}$. Dadurch, dass man dem Dreieck $\Delta$ ein anderes Dreieck einbeschreibt, entstehen innerhalb von $\Delta$ noch drei weitere Dreiecke, die jeweils mit dem Ausgangsdreieck eine Ecke gemeinsam haben. Nach dieser jeweils gemeinsamen Ecke seien diese Eckdreiecke mit $\Delta_{A}, \Delta_{B}$ und $\Delta_{C}$ bezeichnet. Jedes Innendreieck $\Delta_{E}$ erzeugt dementsprechend innerhalb des Ausgangsdreiecks $\Delta$ eine Konfiguration von vier Teildreiecken.

Macht man hinsichtlich der Lage von $X, Y$ und $Z$ gewisse Einschränkungen, so kommt man zu Innendreiecken, über die recht viel bekannt ist. Sind beispielsweise die Transversalen $A X, B Y$ und $C Z$ kopunktal, so ist das Innendreieck ein sogenanntes Ceva-Dreieck,

Einem beliebigen Dreieck $A B C$ kann ein Dreieck $X Y Z$ derart einbeschrieben werden, dass $X, Y$ und $Z$ auf drei verschiedenen Seiten von $A B C$ liegen. Durch diese Konstruktion entsteht eine sehr allgemeine Konfiguration von vier Teildreiecken innerhalb von $A B C$. Die vorliegende Arbeit geht der Frage nach, ob das einbeschriebene Dreieck $X Y Z$ bez. einer metrischen Dreiecksgröße kleiner als die drei übrigen Dreiecke sein kann. Diese Frage wird für die Dreiecksgrößen Fläche, Umfang und Inkreisradius abschlägig beantwortet. Dabei zeigt sich, dass für diese drei Größen eine weitgehend einheitliche Beweismethode verwendet werden kann. Mit dieser Arbeit wird gleichzeitig eine bisher unbewiesene Vermutung von N.D. Kazarinoff aus dem Jahre 1961 bewiesen. 
da für die Konfiguration dann der Satz von CEvA ${ }^{1}$ gilt. Dieser Satz kann in vielfältiger Weise verwendet werden, um Aussagen über besondere Punkte und Geraden im Dreieck zu beweisen (vgl. [2]).

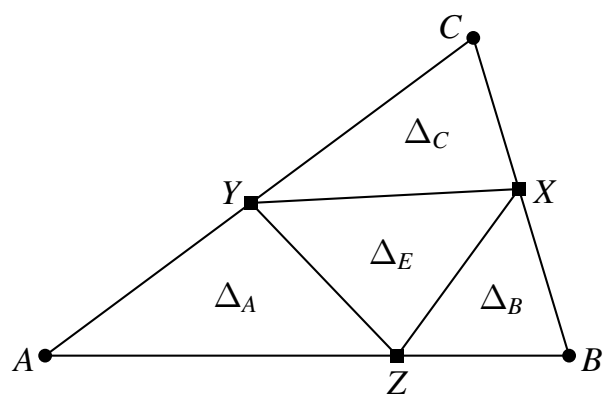

Fig. 1

Ein spezielles Ceva-Dreieck ist das sogenannte Mittendreieck, bei dem $X, Y$ und $Z$ auf den jeweiligen Seitenmitten liegen. Das Mittendreieck führt zu einer Konfiguration, bei der alle vier Teildreiecke untereinander kongruent und gleichzeitig zu $A B C$ ähnlich sind. Diese Konfiguration wird im folgenden Mittenkonfiguration genannt.

In dieser Arbeit sollen jedoch für die Lage der Punkte $X, Y$ und $Z$ keinerlei Einschränkungen gemacht werden, so dass ein Rückgriff auf den Satz von Ceva ausgeschlossen ist. Über in diesem Sinne sehr allgemeine Konfigurationen ist recht wenig bekannt, vermutlich weil es auch nur wenige gemeinsame Eigenschaften gibt. Eine Ausnahme bildet hier der Satz von MIQUEL ${ }^{2}$, der besagt, dass sich bei jeder Konfiguration die Umkreise der drei Eckdreiecke in einem gemeinsamen Punkt $M$ schneiden. Über den Miquelschen Punkt $M$ selbst und das zugehörige Innendreieck gibt es weitere sehr interessante Folgerungen, die DonATH ([2, S. 44ff]) zusammenfasst. Im Laufe dieser Arbeit wird aber auf den Miquelschen Satz nicht zurückgegriffen.

\section{Die Vermutung von Kazarinoff}

KAZARINOFF ([3, S. 84]) listet 1961 am Ende seines Buches Analytic Inequalities einige ungelöste Vermutungen auf. Er schreibt u.a.

Wenn man einem gegebenen Dreieck ein anderes einbeschreibt, wird es dadurch in vier kleinere Dreiecke zerlegt.

(a) Das einbeschriebene Dreieck hat niemals die kleinste Fläche der vier Teildreiecke. (Hinweis: Finde eine affine Abbildung)

(b) (Vermutung) Das einbeschriebene Dreieck hat niemals den kleinsten Umfang der vier Teildreiecke.

\footnotetext{
${ }^{1}$ Giovanni Ceva (1647?-1734), italienischer Mathematiker

${ }^{2}$ Auguste Miquel, französischer Mathematiker (Der Satz wurde 1838 veröffentlicht.)
} 
OGILVY ([4, S. 39]) nimmt 1962 die Vermutung von Kazarinoff in seine bekannte Sammlung ungelöster Probleme auf.

Man beachte, dass hier nicht nach einem absoluten Minimum gefragt wird, d.h. es handelt sich bei Kazarinoffs Problem nicht um das berühmte Problem von FAGNANO-SCHWARZ ${ }^{3}$, das nach dem Innendreieck mit dem absolut minimalen Umfang sucht. Die Lösung dieses Problems ist im spitzwinkligen Dreieck bekanntlich das Höhenfußpunktdreieck. Beim Höhenfußpunktdreieck ist eines der verbleibenden Eckdreiecke allerdings immer von geringerem Umfang als das Höhenfußpunktdreieck selbst. Es scheidet daher als Lösung des Kazarinoff-Problems aus, denn hier wird nach einem relativ-minimalen Innendreieck gefragt, das nicht notwendigerweise mit dem absolut-minimalen Innendreieck identisch sein muss.

Wie sich zeigen wird, hängt die Antwort auf die Frage von Kazarinoff davon ab, mit welchem Maß man die Größe der beteiligten Dreiecke misst. Die Möglichkeiten für Maßgrößen bei Dreiecken sind recht vielfältig. Kazarinoff erwähnt selbst schon neben dem Umfang auch die Fläche als Maßgröße. Weitere übliche Größen sind der Inkreisradius und der Umkreisradius, denkbar wären aber auch das Seitenprodukt, die Summe der Ankreisradien und viele andere mehr. Solche Maßgrößen $\mu$ müssen sich letztendlich immer als symmetrische Funktionen der drei Dreiecksseiten schreiben lassen, d.h. der Funktionsterm zu $\mu(a, b, c)$ muss invariant gegenüber einer zyklischen Vertauschung der Variablen $a, b$ und $c$ sein. Derartige Maßgrößen für das Dreieck werden im nachfolgenden Text als metrische Dreiecksgrößen $\mu$ bezeichnet.

Nach diesen Erläuterungen kann das verallgemeinerte Kazarinoff-Problem genauer formuliert werden. Die Bearbeitung der Vermutung von Kazarinoff stellt sich dann als Suche nach einer $\mu$-minimalen Konfiguration dar.

Das verallgemeinerte Kazarinoff-Problem. Gibt es eine Konfiguration, bei der das Innendreieck bez. einer metrischen Dreiecksgröße $\mu$ kleiner als jedes der drei Eckdreiecke ist? Eine Konfiguration, die diese Forderung erfüllt, nennen wir eine $\mu$-minimale Konfiguration.

\section{Ein Beispiel einer $r$-minimalen Konfiguration}

Misst man die Größe der Dreiecke über den Umkreisradius $r$, so kann man an einem konkreten Beispiel sehen, dass es tatsächlich $r$-minimale Konfigurationen gibt. Dazu gehe man von einem gleichseitigen Innendreieck $\Delta_{E}$ mit der Seitenlänge 2 aus, dem man ein Dreieck $\Delta$ umschreibt, wie es Fig. 2 zeigt. Für den Umkreisradius $r$ gilt die bekannte Gleichung

$$
r=\frac{a}{2 \sin \alpha}=\frac{b}{2 \sin \beta}=\frac{c}{2 \sin \gamma}
$$

\footnotetext{
${ }^{3}$ Giovanni Francesco Fagnano (1715-1779), italienischer Mathematiker; Hermann Amandus Schwarz (18431921), deutscher Mathematiker (Das Problem wurde 1775 von Fagnano gestellt, Schwarz fand eine elegante und elementare Lösung.)
} 


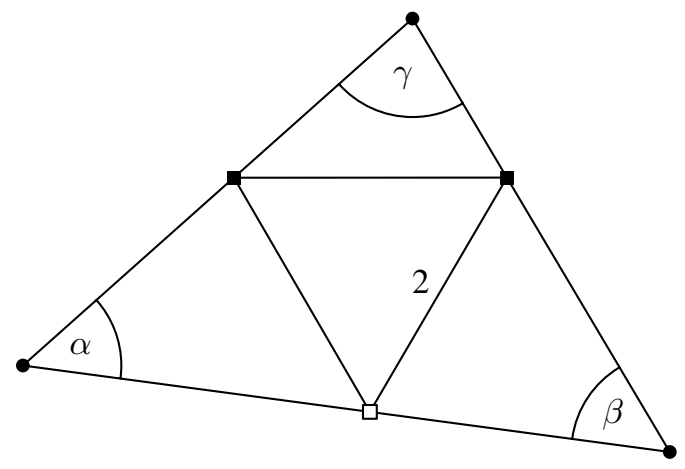

Fig. 2

Bezeichnet man nun die Umkreisradien der vier Teildreiecke mit $r_{A}, r_{B}, r_{C}$ und $r_{E}$, so ergeben sich die Werte

$$
r_{A}=\frac{1}{\sin \alpha}, \quad r_{B}=\frac{1}{\sin \beta}, \quad r_{C}=\frac{1}{\sin \gamma}, \quad r_{E}=\frac{1}{\sin 60^{\circ}} .
$$

Bei der Konstruktion des umschriebenen Dreiecks $\Delta$ muss man also lediglich darauf achten, dass die Sinuswerte der drei Eckwinkel alle kleiner als $\sin 60^{\circ}$ sind. Dies ist zum Beispiel für $\alpha=\beta=20^{\circ}$ und $\gamma=140^{\circ}$ der Fall.

Damit ist klar, dass das verallgemeinerte Kazarinoff-Problem für die metrische Größe $r$ lösbar ist. Es sei allerdings noch darauf hingewiesen, dass für gewisse Dreiecke $\Delta$ keine Lösung möglich ist. Hat $\Delta$ beispielsweise einen rechten Winkel bei $\alpha$, so kann der Umkreisradius von $\Delta_{E}$ nie kleiner werden als der von $\Delta_{A}$.

\section{Monotone metrische Dreiecksgrößen}

Im weiteren Verlauf dieser Arbeit wird gezeigt werden, dass für die übrigen gebräuchlichen metrischen Größen Fläche $F$, Umfang $U$ und Inkreisradius $\rho$ die Vermutung von Kazarinoff richtig ist, dass es also für diese Größen keine minimale Konfiguration geben kann. Entscheidend dafür ist, dass diese Größen in einem gewissen Sinne monoton sind, was für den Umkreisradius nicht der Fall ist. Um den Begriff der Monotonie zu präzisieren, gehe man von einer Situation aus, die in Fig. 3 dargestellt ist. Bewegt man hier den Punkt $A^{\prime}$ auf der Seite $A C$, so ändert sich die Form von $A^{\prime} B C$ stetig. Wie verhält es sich dabei mit der Änderung der metrischen Größe $\mu\left(A^{\prime} B C\right)$ ? Ist die Messgröße beispielsweise die Fläche, so wird diese um so kleiner, je näher $A^{\prime}$ an $C$ heranrückt. Dasselbe gilt für den Umfang und den Inkreisradius, da das Dreieck $A^{\prime} B C$ bei diesem Prozess immer vollständig innerhalb von $A B C$ liegt. Die Veränderung dieser Messgrößen ist also monoton. Beim Umkreisradius ist dies aber nicht der Fall, denn hier erreicht $\mu\left(A^{\prime} B C\right)$ ein Minimum, wenn der Winkel bei $A^{\prime}$ rechtwinklig ist.

Definition Unter einer monotonen metrischen Dreiecksgröße $\mu$ verstehen wir eine Messgröße beim Dreieck, die folgende Eigenschaft erfüllt: Ist $\Delta_{1}$ ein Dreieck, das ganz in einem Dreieck $\Delta_{2}$ enthalten ist, so gilt $\mu\left(\Delta_{1}\right)<\mu\left(\Delta_{2}\right)$. 


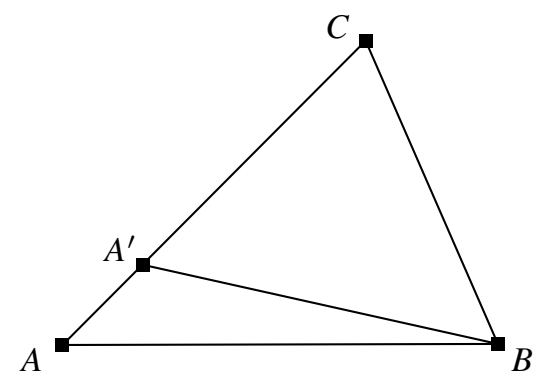

Fig. 3

Beim Beweis des Hauptsatzes über minimale Konfigurationen spielt die Monotonie der Messfunktion eine entscheidende Rolle. Ein weiteres Hilfsmittel bei den späteren Betrachtungen sind ganz besondere Konfigurationen, die im nächsten Abschnitt erläutert werden.

\section{Isometrische Konfigurationen}

Als besonderes Innendreieck einer Konfiguration wurde eingangs schon das Mittendreieck erwähnt. Wegen der Kongruenz der vier Teildreiecke in der Mittenkonfiguration, sind diese auch bez. jeder metrischen Dreiecksgröße gleich. Es ergibt sich daher die Frage, ob es andere Konfigurationen gibt, bei der die vier Teildreiecke wenigstens hinsichtlich einer metrischen Größe gleich sind.

Definition Eine Konfiguration heißt $\mu$-isometrisch, wenn die vier Teildreiecke bez. der metrischen Größe $\mu$ denselben Wert haben. Eine von der Mittenkonfiguration verschiedene $\mu$-isometrische Konfiguration nennen wir eine nicht-triviale $\mu$-isometrische Konfguration.

Wir werden sehen, dass $\mu$-isometrische Konfigurationen sehr seltene Exemplare sind, dass es für etliche metrische Größen $\mu$ außer der Mittenkonfiguration gar keine weiteren gibt. Um so erfreulicher ist es, dass es hinsichtlich des Umkreisradius $r$ tatsächlich solche $r$ isometrischen Konfigurationen gibt. Um ein Beispiel für ein solches Dreieck zu konstruieren, nehme man als Ausgangsdreieck ein gleichseitiges Dreieck $A B C$. Auf den Seiten dieses Dreiecks verteile man die Punkte $X, Y$ und $Z$ so, dass die Seiten $a, b$ und $c$ im Uhrzeigersinn alle im gleichen Verhältnis geteilt werden (vgl. Fig. 4). Man sieht dann leicht, dass die drei entstandenen Eckdreiecke untereinander kongruent sind, da sie in zwei Seiten und dem eingeschlossenen $60^{\circ}$-Winkel übereinstimmen. Demnach sind jedenfalls die Umkreise der drei Eckdreiecke gleich. Die Kongruenz der drei Eckdreiecke führt aber auch dazu, dass das Innendreieck gleichseitig ist. Demnach sind die Winkel bei $A$ und bei $X$ beide $60^{\circ}$ groß. Diese Winkel sind aber beide Peripheriewinkel über derselben Sehne $Y Z$. Als gleich große Peripheriewinkel müssen sie zum selben Kreis gehören, woraus sofort folgt, dass die Umkreise von $\Delta_{A}$ und $\Delta_{E}$ gleich sind. Die Konfiguration ist demnach $r$-isometrisch und liefert natürlich auch nicht-triviale Lösungen.

In der Mittenkonfiguration werden die Dreiecksseiten $a, b$ und $c$ in jeweils gleich große Abschnitte geteilt. Drei solcher Abschnitte, von denen je zwei keinen gemeinsamen Punkt 


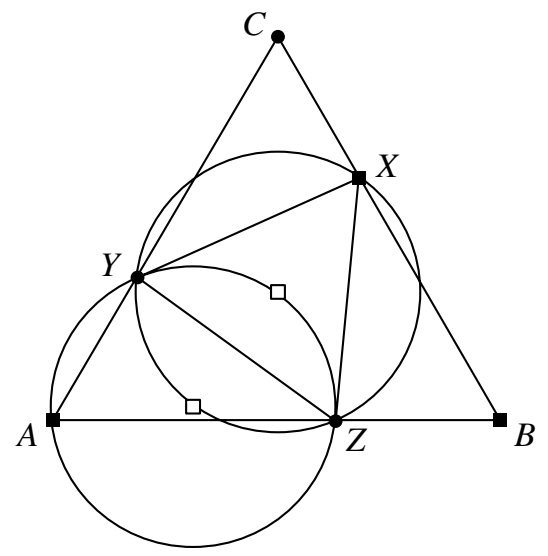

Fig. 4

haben, nennt man alternierende Abschnitte. Das eben konstruierte Beispiel einer isometrischen Konfiguration zeigt, dass hier die Eckpunkte $X, Y$ und $Z$ in alternierenden Abschnitten liegen. Der folgende Satz verallgemeinert diese Beobachtung für monotone metrische Größen.

Satz 1 Ist $\mu$ eine monotone metrische Dreiecksgröße, so müssen bei einer $\mu$-isometrischen Konfiguration die Eckpunkte $X, Y$ und $Z$ des Innendreiecks $\Delta_{E}$ in alternierenden Abschnitten von $\Delta$ liegen. Bei einer nicht-trivialen isometrischen Konfiguration kann keiner der Punkte $X, Y$ und $Z$ Seitenmittelpunkt zu $\Delta$ sein.

Beweis. Läge einer der Eckpunkte von $\Delta_{E}$ (o.B.d.A. sei dies $X$ ) auf einer Seitenmitte und ein anderer nicht (hier $Y$ ), so läge $C M_{b} M_{a}$ innerhalb von $C Y X$, d.h. $\mu\left(C M_{b} M_{a}\right)$ wäre kleiner als $\mu(C Y X)$. Andererseits wäre entweder $\mu(A Z Y)$ oder $\mu(B X Z)$ wegen der Monotonie kleiner als der $\mu$-Wert des entsprechenden Eckdreiecks der Mittenkonfiguration (hier o.B.d.A. $B X Z$ ). Die $\mu$-Werte von $\Delta_{C}$ und $\Delta_{B}$ könnten also nicht gleich sein, da zwischen ihnen der $\mu$-Wert der Mittenkonfiguration läge. Aus $X=M_{a}$ würde also sofort folgen, dass $\Delta_{E}$ das Mittendreieck sein müsste. Läge $X$ sogar innerhalb der Strecke $M_{a} B$, so würde mit demselben Argument folgen, dass die $\mu$-Werte zweier Eckdreiecke nicht übereinstimmen könnten, da sie vom $\mu$-Wert der Mittenkonfiguration getrennt würden. Ist also eine isometrische Konfiguration verschieden von der Mittenkonfiguration, so müssen die Eckpunkte $X, Y$ und $Z$ echt innerhalb von alternierenden Abschnitten liegen.

Angemerkt sei noch, dass der Satz 1 in Verbindung mit dem Satz von Ceva sofort zu der nachstehenden Ergänzung führt, da bei einer Ceva-Konfiguration die Produkte der alternierenden Abschnitte gleich sein müssen. Bei einer isometrischen Konfiguration besteht aber die eine Gruppe alternierender Abschnitte immer aus den längeren Teilstücken der Seite.

Korollar zu Satz 1 Die Mittenkonfiguration ist für monotone $\mu$-Größen die einzige $\mu$ isometrische Ceva-Konfiguration. 


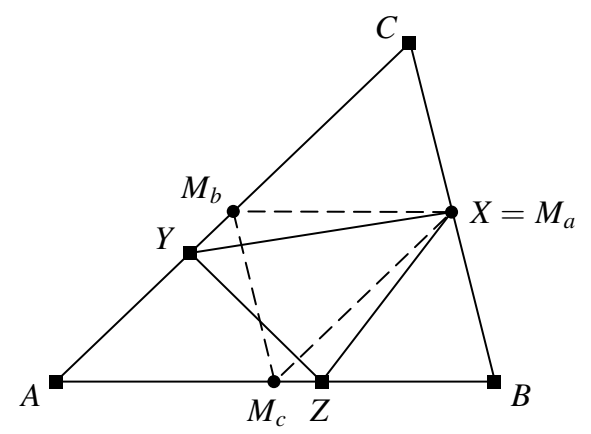

Fig. 5

Im nachfolgenden Abschnitt werden wir sehen, dass die Existenz von minimalen Konfigurationen sehr eng mit der Existenz von nicht-trivialen isometrischen Konfigurationen verknüpft ist.

\section{Der Hauptsatz über minimale Konfigurationen}

Zunächst benötigen wir einen Satz über das Mittendreieck, der gewissermaßen ein hinreichendes Kriterium für das Mittendreieck liefert. Später werden wir übrigens sehen, dass aus der Lösung der Kazarinoff-Vermutung weitere hinreichende Kriterien für das Mittendreieck gewissermaßen als Nebenprodukte abfallen.

Satz 2 (Hinreichendes Kriterium für das Mittendreieck) Die Mittenkonfiguration ist die einzige Konfiguration, bei der die Eckdreiecke zu $\Delta$ gleichsinnig-ähnlich sind.

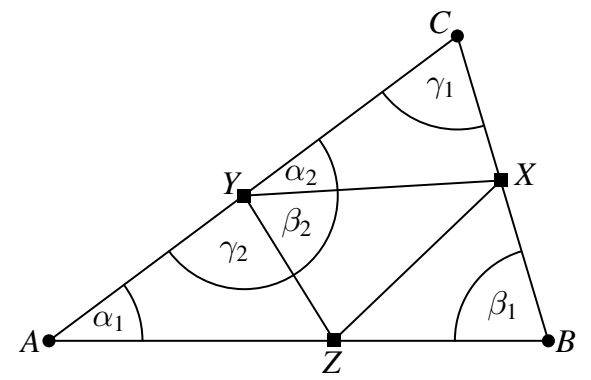

Fig. 6

Beweis. Wegen der Ähnlichkeit der Eckdreiecke ist sofort klar, dass in Fig. 6 alle $\alpha$-Winkel in den Eckdreiecken übereinstimmen müssen. Dasselbe gilt natürlich für die $\beta$ - und $\gamma-$ Winkel in den Eckdreiecken. Für das Innendreieck bleiben dann als Ergänzung auf $180^{\circ}$ jeweils nur ein $\alpha$-, ein $\beta$ - und ein $\gamma$-Winkel, d.h. $\Delta_{E}$ ist ebenfalls zu $\Delta$ ähnlich. Da das Innendreieck aber mit jedem Eckdreieck eine gemeinsame Seite hat, sind die vier Teildreiecke sogar kongruent zueinander. Dann müssen aber auch die Teilstücke $C X$ und $X B$ gleich sein, d.h. $\Delta_{E}$ ist das Mittendreieck. 
Es folgt nun der

Hauptsatz über minimale Konfigurationen Es sei $\mu$ eine monotone metrische Dreiecksgröße. Es kann nur dann eine $\mu$-minimale Konfiguration geben, wenn es auch eine nichttriviale $\mu$-isometrische Konfiguration gibt.

Beweis. Es sei also angenommen, dass $\Delta_{E}$ in der Fig. 7 ein Innendreieck einer $\mu$-minimalen Konfiguration ist. Dann kann $\Delta_{E}$ natürlich kein Mittendreieck sein. Nach Satz 2 ist dann mindestens eines der drei Eckdreiecke nicht zu $\Delta$ ähnlich. Es sei dies o.B.d.A. $\Delta_{A}$. Daraus folgt weiter, dass dann entweder $\beta$ kleiner als $\beta_{1}$ oder $\gamma$ kleiner als $\gamma_{1}$ sein muss. Wir nehmen wiederum o.B.d.A. an, dass $\beta<\beta_{1}$ ist.

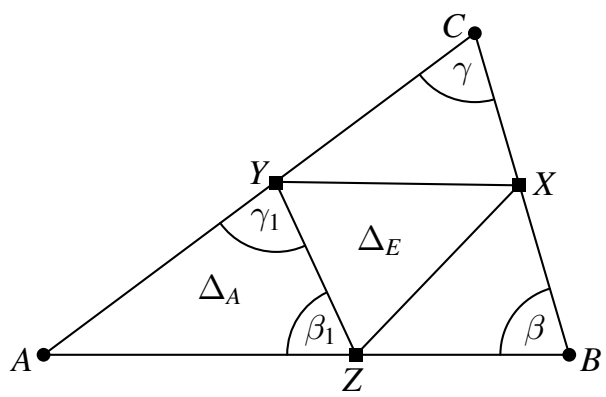

Fig. 7

Nun wird eine monoton wachsende Folge von Winkeln $\beta_{1}, \beta_{2}, \beta_{3}, \ldots$ konstruiert. Dazu drehe man die Seite $A B$ um den Punkt $Z$ im mathematisch positiven Sinn. Der Punkt $A$ wandert bei dieser Drehung auf der Verlängerung von $C A$, der Punkt $B$ wandert entsprechend auf der Seite $B C$. Durch diese Drehung ist das Eckdreieck $\Delta_{A}$ relativ zu $\Delta_{E}$ vergrößert worden, wohingegen $\Delta_{B}$ relativ verkleinert wurde. Das neue Eckdreieck $\Delta_{B}$ liegt komplett innerhalb des alten $\Delta_{B}$, d.h. der $\mu$-Wert wurde verkleinert. Die Drehung soll allerdings nur so weit gehen, bis $\mu\left(\Delta_{B}\right)=\mu\left(\Delta_{E}\right)$ ist.

Nun führt man die entsprechende Drehung mit der Seite $B C$ um den Punkt $X$ herum aus. Der $\mu$-Wert von $\Delta_{C}$ wird mit dem von $\Delta_{E}$ in Übereinstimmung gebracht. Bei diesem Prozess vergrößert sich natürlich wieder $\mu\left(\Delta_{B}\right)$. Im dritten Schritt wird nun die Drehung von $C A$ um $Y$ im selben Sinne durchgeführt. Nach diesen drei Drehungen gilt

$$
\mu\left(\Delta_{A}\right)=\mu\left(\Delta_{E}\right), \quad \mu\left(\Delta_{B}\right)>\mu\left(\Delta_{E}\right), \quad \mu\left(\Delta_{C}\right)>\mu\left(\Delta_{E}\right) .
$$

Aus dem Winkel $\beta_{1}$ ist allerdings ein größerer Winkel $\beta_{2}$ geworden.

Da $\Delta_{B}$ nun wieder größer als $\Delta_{E}$ ist, können wir den Prozess fortsetzen, um zu einem Winkel $\beta_{3}$ zu gelangen. Insgesamt erzeugen wir so eine streng monoton steigende Folge von Winkeln

$$
\beta<\beta_{1}<\beta_{2}<\beta_{3}<\ldots
$$

am Punkt $Z$. Diese Folge ist aber nach oben beschränkt, denn der $\beta$-Winkel bei $Z$ kann nie größer als $180^{\circ}-\gamma_{E}$ werden, wobei $\gamma_{E}$ der Winkel von $\Delta_{E}$ bei $Z$ ist. Als monoton wach- 


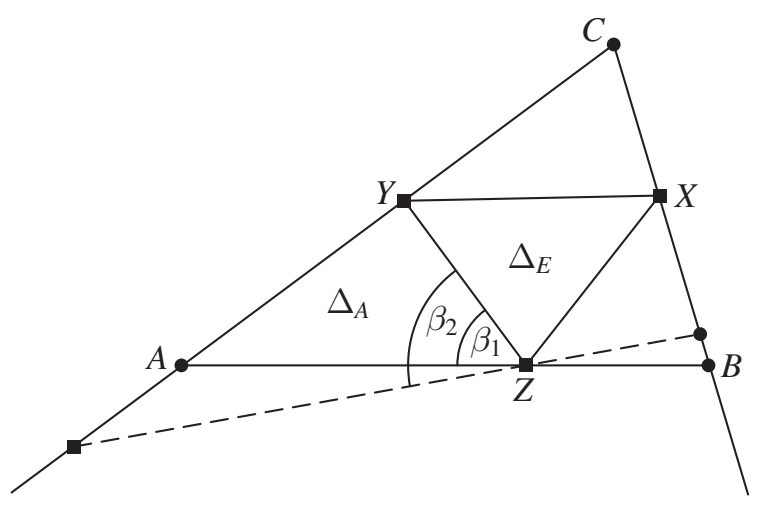

Fig. 8

sende und nach oben beschränkte Folge muss die Folge der $\beta$-Winkel einen Grenzwert haben. Beim Erreichen dieses Grenzwertes kann eine Fortsetzung des Drehprozesses nicht mehr möglich sein, da man ansonsten den $\beta$-Winkel weiter vergrößern könnte. Wenn der Drehprozess zum Stillstand kommt, kann das nur bedeuten, dass alle drei Eckdreiecke im $\mu$-Wert mit $\Delta_{E}$ übereinstimmen, also auch untereinander übereinstimmen. Wir haben also eine $\mu$-isometrische Konfiguration erzeugt. Da aber mindestens das Eckdreieck $\Delta_{A}$ wegen des $\beta$-Winkels nicht zu $\Delta$ ähnlich ist, kann diese Konfiguration nicht die Mittenkonfiguration sein, d.h. wir haben sogar eine nicht-triviale $\mu$-isometrische Konfiguration erzeugt.

\section{Anmerkungen zur Beweistechnik}

Für die monotonen metrischen Dreiecksgrößen $F, U$ und $\rho$ soll nun das KazarinoffProblem dadurch gelöst werden, dass wir die Existenz von nicht-trivialen isometrischen Konfigurationen untersuchen. Die Methode in diesen Beweisen ist sehr einheitlich und lässt sich vermutlich auf andere monotone metrische Dreiecksgrößen, die hier nicht untersucht werden, übertragen. Bei aller Ähnlichkeit der Beweise verlangt allerdings jede metrische Größe noch ihre eigene zusätzliche Idee.

Alle Beweise werden indirekt geführt, d.h. wir nehmen jeweils an, dass es eine entsprechende nicht-triviale $\mu$-isometrische Konfiguration gibt und führen dies zum Widerspruch.

Wir können also nach Satz 1 jeweils annehmen, dass keiner der Punkte $X, Y$ und $Z$ auf einer Seitenmitte von $\Delta$ liegt. Da diese Punkte obendrein in alternierenden Abschnitten liegen müssen, kann man ferner annehmen, dass die im mathematisch positiven Sinne ersten Abschnitte die jeweils größeren sind. Da demnach die Punkte $X, Y$ und $Z$ jenseits der Seitenmitte liegen, legen wir folgende Abkürzungen fest, die in allen Beweisen zur Anwendung kommen:

$$
\begin{aligned}
& a_{1}=\left(\frac{1}{2}+a^{*}\right) \cdot a, \quad b_{1}=\left(\frac{1}{2}+b^{*}\right) \cdot b, \quad c_{1}=\left(\frac{1}{2}+c^{*}\right) \cdot c, \\
& a_{2}=\left(\frac{1}{2}-a^{*}\right) \cdot a, \quad b_{2}=\left(\frac{1}{2}-b^{*}\right) \cdot b, \quad c_{2}=\left(\frac{1}{2}-c^{*}\right) \cdot c .
\end{aligned}
$$




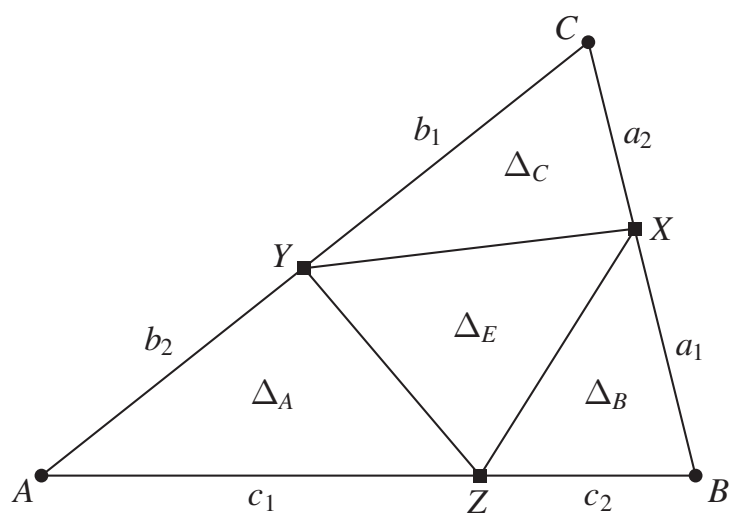

Fig. 9

Man beachte, dass die Sterngrößen hierbei alle positiv sein müssen. Die Verwendung dieser Sterngrößen wird sich als der entscheidende Schlüssel für die Herleitung des Widerspruchs zeigen.

\section{$F$-minimale Konfigurationen}

Wir beginnen mit der metrischen Dreiecksgröße $F$ für den Flächeninhalt, da hier der Beweis recht kurz ausfällt und dennoch die grundsätzliche Beweistechnik deutlich wird.

Wir lösen diesen Fall für das allgemeine Dreieck, obgleich es wegen der Flächenverhältnistreue der affinen Abbildungen genügen würde, diesen Fall für gleichseitige Dreiecke zu behandeln. Der Gewinn hinsichtlich der Kürze des Beweises wäre allerdings nur minimal, dafür würde aber die Vergleichbarkeit der Beweisführungen für die nachfolgenden Sätze verloren gehen. Gerade dieser Aspekt ist aber wichtig, da die Beweise der weiteren Fälle besser nachvollziehbar sind, wenn man an diesem einfachen Fall die Struktur des Beweisaufbaus erkennt.

Satz 3 Es gibt keine nicht-triviale F-isometrische Konfiguration, also auch keine Fminimale-Konfiguration.

Beweis. Aus der allgemeinen Konfigurationsfigur (Fig. 1) entnimmt man sofort die Gleichung

$$
F_{A}+F_{B}+F_{C}+F_{E}=F \text {. }
$$

Bei einer $F$-isometrischen Konfiguration muss also jedes der Teildreiecke ein Viertel der Gesamtfläche ausmachen.

Wie üblich sei nun mit $\alpha$ der Innenwinkel von $\Delta$ bei $A$ gemeint. Da $\Delta_{A}$ ein Viertel der Fläche von $\Delta$ hat, ergibt sich mit der üblichen Flächenformel für das Dreieck:

$$
\begin{aligned}
& \frac{1}{2}\left(\frac{1}{2}-b^{*}\right) \cdot b \cdot\left(\frac{1}{2}+c^{*}\right) \cdot c \cdot \sin \alpha=\frac{1}{4} \cdot \frac{1}{2} \cdot b \cdot c \cdot \sin \alpha \\
& \Longleftrightarrow\left(\frac{1}{2}-b^{*}\right) \cdot\left(\frac{1}{2}+c^{*}\right)=\frac{1}{4} \Longleftrightarrow\left(1-2 b^{*}\right) \cdot\left(1+2 c^{*}\right)=1 .
\end{aligned}
$$


Multipliziert man die Klammern aus, so ergibt sich die Beziehung:

$$
\Longleftrightarrow-2 b^{*}+2 c^{*}=4 b^{*} c^{*} \Longleftrightarrow-b^{*}+c^{*}=2 b^{*} c^{*} \quad\left(\Delta_{A}\right) \text {. }
$$

Da für die beiden anderen Eckdreiecke dieselben Bedingungen herrschen, braucht man nur eine zyklische Vertauschung der Variablen vorzunehmen, um die entsprechenden Beziehungen für die beiden anderen Eckdreiecke zu erhalten:

$$
\begin{aligned}
& -c^{*}+a^{*}=2 c^{*} a^{*} \quad\left(\Delta_{B}\right) \\
& -a^{*}+b^{*}=2 a^{*} b^{*} \quad\left(\Delta_{C}\right) \text {. }
\end{aligned}
$$

Addiert man die drei Gleichungen, so ergibt sich

$$
0=2\left(b^{*} c^{*}+c^{*} a^{*}+a^{*} b^{*}\right) .
$$

Diese Gleichung muss falsch sein, da alle Sterngrößen positiv sind. Damit ist die Annahme der Existenz einer nicht-trivialen $F$-isometrischen Konfiguration zum Widerspruch geführt.

Nebenbei sei darauf hingewiesen, dass man den Satz 3 auch als hinreichendes Kriterium für das Mittendreieck interpretieren kann. Aus der Flächengleichheit der Teildreiecke, sogar schon der Eckdreiecke, folgt, dass die Konfiguration die Mittenkonfiguration sein muss. Eine entsprechende Interpretation ist natürlich auch für die folgenden Sätze möglich.

\section{$U$-minimale Konfigurationen}

Der nächste Satz löst das ursprünglich von Kazarinoff formulierte Problem für den Umfang $U$ der Teildreiecke. Im Beweis tritt hier an die Stelle der Flächenformel der Kosinussatz der Trigonometrie. Allerdings sind die notwendigen Termumformungen erheblich aufwändiger und weniger naheliegend als beim Fall der Flächen.

Satz 4 Es gibt keine nicht-triviale $U$-isometrische Konfiguration, also auch keine $U$ minimale Konfiguration.

Beweis. Wir nehmen wiederum die Existenz einer nicht-trivialen $U$-isometrischen Konfiguration an. Aus der allgemeinen Konfigurationsfigur (Fig. 1) entnimmt man zunächst die Gleichung

$$
U_{A}+U_{B}+U_{C}=U+U_{E}
$$

Ersetzt man alle Teilumfänge durch $U^{*}$, so erhält man

$$
2 U^{*}=U \text {. }
$$

Bei einer $U$-isometrischen Konfiguration muss also jedes der Teildreiecke die Hälfte des Umfangs von $\Delta$ ausmachen. 
Nun formen wir den Kosinussatz zu einer Formel für den Umfang von $\Delta$ um, die dann an die Stelle der Flächenformel im vorigen Beweis tritt.

$$
\begin{aligned}
& a^{2}=b^{2}+c^{2}-2 b c \cos \alpha \\
& \Longleftrightarrow 2 b c \cos \alpha+2 b c=b^{2}+2 b c+c^{2}-a^{2} \\
& \Longleftrightarrow 2 b c(1+\cos \alpha)=(b+c)^{2}-a^{2}=(b+c-a)(b+c+a) \\
& \Longleftrightarrow 2 b c(1+\cos \alpha)=(b+c-a) U .
\end{aligned}
$$

Für die Klammer der rechten Seite ergibt sich mit der üblichen Bezeichnung $s$ für den halben Dreiecksumfang

$$
b+c-a=2 s-2 a=2(s-a) .
$$

Setzt man dies in der Zeile (3) ein, so erhält man

$$
U=\frac{b c(1+\cos \alpha)}{s-a}
$$

Da das Eckdreieck $\Delta_{A}$ den halben Umfang von $\Delta$ hat, liefert diese Formel die Gleichung

$$
\begin{aligned}
& \frac{\left(\frac{1}{2}-b^{*}\right) \cdot b \cdot\left(\frac{1}{2}+c^{*}\right) \cdot c \cdot(1+\cos \alpha)}{\frac{s}{2}-x}=\frac{1}{2} \cdot \frac{b c(1+\cos \alpha)}{s-a} \\
& \Longleftrightarrow\left(\frac{1}{2}-b^{*}\right) \cdot\left(\frac{1}{2}+c^{*}\right)=\frac{1}{2} \cdot \frac{\frac{s}{2}-x}{s-a} .
\end{aligned}
$$

Beidseitige Multiplikation mit 4 führt zu

$$
\left(1-2 b^{*}\right)\left(1+2 c^{*}\right)=\frac{s-2 x}{s-a} .
$$

Damit sind wir an einer Stelle angelangt, die der Zeile (1) im vorigen Beweis entspricht. Im Term der rechten Seite muss allerdings noch die Seitenlänge $x$ des Innendreiecks eliminiert werden. Es gilt

$$
\begin{aligned}
s-2 x & =s-2\left(U_{E}-b_{2}-c_{1}\right)=s-2\left(s-\left(\frac{1}{2}-b^{*}\right) b-\left(\frac{1}{2}+c^{*}\right) c\right) \\
& =-s+b-2 b^{*} b+c+2 c^{*} c=s-a-2 b^{*} b+2 c^{*} c .
\end{aligned}
$$

Setzt man dies in (4) ein und löst gleichzeitig die Klammern der linken Seite von (4) auf, so erhält man

$$
\begin{aligned}
& 1-2 b^{*}+2 c^{*}-4 b^{*} c^{*}=1-\frac{2 b^{*} b-2 c^{*} c}{s-a} \\
& \Longleftrightarrow-2 b^{*}(s-a)+2 c^{*}(s-a)-4 b^{*} c^{*}(s-a)=-2 b^{*} b+2 c^{*} c \\
& \Longleftrightarrow b^{*}(-s+a+b)-c^{*}(-s+a+c)=2 b^{*} c^{*}(s-a) \\
& \Longleftrightarrow b^{*}(s-c)-c^{*}(s-b)=2 b^{*} c^{*}(s-a) .
\end{aligned}
$$


Erweitern wir diese Gleichung noch beidseitig mit $a^{*}$, so erhalten wir damit ein Analogon zur Zeile (2) im vorigen Beweis

$$
\Longleftrightarrow a^{*} b^{*}(s-c)-a^{*} c^{*}(s-b)=2 a^{*} b^{*} c^{*}(s-a) \quad\left(\Delta_{A}\right) .
$$

Durch zyklische Vertauschung der Variablen ergeben sich wiederum die entsprechenden Gleichungen für die beiden anderen Eckdreiecke:

$$
\begin{array}{lll}
b^{*} c^{*}(s-a)-b^{*} a^{*}(s-c) & =2 a^{*} b^{*} c^{*}(s-b) & \left(\Delta_{B}\right), \\
c^{*} a^{*}(s-b)-c^{*} b^{*}(s-a) & =2 a^{*} b^{*} c^{*}(s-c) & \left(\Delta_{C}\right) .
\end{array}
$$

Die Addition der drei Gleichungen liefert wieder den gesuchten Widerspruch

$$
0=2 a^{*} b^{*} c^{*}(s-a+s-b+s-c),
$$

denn alle Faktoren der rechten Seite sind positiv. Damit ist die Nichtexistenz von $U$ minimalen Konfigurationen nachgewiesen.

\section{Anmerkungen zur Beweisstruktur}

Im Vergleich zum Flächenproblem sind die beim Umfangsproblem durchgeführten Termumformungen recht aufwändig. Die Beweisstruktur ist allerdings in beiden Fällen völlig vergleichbar. Man kann folgende sechs Beweisschritte erkennen:

1. Feststellen des Verhältnisses $\mu\left(\Delta_{A}\right) / \mu(\Delta)$ bei $\mu$-Isometrie

2. Anwendung einer Formel für $\mu$ auf $\Delta$ und $\Delta_{A}$

3. Herleitung einer Beziehung der Sterngrößen für das Eckdreieck $\Delta_{A}$

4. Zyklische Ergänzung der entsprechenden Beziehungen für $\Delta_{B}$ und $\Delta_{C}$

5. Addition dieser drei Beziehungen

6. Widerspruch zur Lage der Eckpunkte $X, Y$ und $Z$ in einer nicht-trivialen isometrischen Konfiguration

Genau diese sechs Beweisschritte werden auch im nächsten Fall durchgeführt werden, wobei sich dort aber herausstellt, dass bei den Radien der Inkreise der erste Beweisschritt der aufwändigste Schritt ist, während er sich in den beiden schon behandelten Fällen sofort aus der Anschauung (Fig. 1) ergab.

\section{$\rho$-minimale Konfigurationen}

Die Durchführung des ersten Beweisschrittes wird der Übersichtlichkeit halber auf drei Hilfssätze verteilt. Bei den Beweisen dieser Hilfssätze kommt schon die Formel zum Einsatz, die auch die Grundlage für den zweiten Beweisschritt darstellt. Es handelt sich diesmal um die Formel, die den Zusammenhang zwischen Fläche, Umfang und Inkreisradius beschreibt:

$$
F=\rho \cdot s
$$




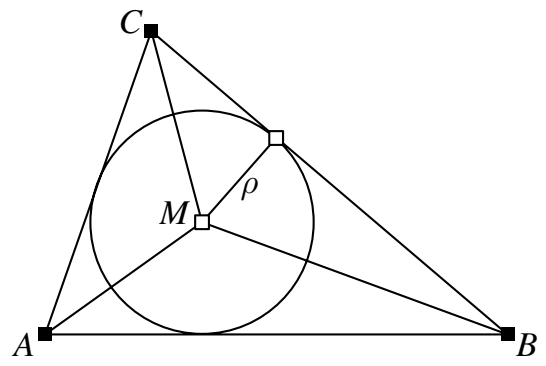

Fig. 10

Sie ergibt sich bekanntlich aus Fig. 10, wenn man die Fläche von $A B C$ als Summe der Teilflächen $A B M, B C M$ und $C A M$ ausdrückt.

In einer Konfiguration (Fig. 1) wird diese Formel zu

$$
F_{A}+F_{B}+F_{C}+F_{E}=\rho \cdot s .
$$

In einer $\rho$-isometrischen Konfiguration mit dem gemeinsamen Inkreisradius $\rho^{*}$ kann man die Teilflächen der linken Seite derselben Formel entsprechend ersetzen:

$$
\rho^{*} s_{A}+\rho^{*} s_{B}+\rho^{*} s_{C}+\rho^{*} s_{E}=\rho \cdot s .
$$

Aus der Summenformel der Umfänge in einer Konfiguration

$$
U_{A}+U_{B}+U_{C}=U+U_{E}
$$

ergibt sich die analoge Formel für die halben Umfänge, so dass (6) zu

$$
\rho^{*}\left(s+s_{E}+s_{E}\right)=\rho \cdot s
$$

wird. Daraus ergibt sich der erste Hilfssatz:

Hilfssatz 1 In einer $\rho$-isometrischen Konfiguration mit dem gemeinsamen Inkreisradius $\rho^{*}$ gilt

$$
\frac{\rho}{\rho^{*}}=1+\frac{2 s_{E}}{s}
$$

Damit ist das Verhältnis der Inkreisradien in einer $\rho$-isometrischen Konfiguration in einer ersten Form beschrieben. Man kann es aber auch in anderer Form beschreiben:

Hilfssatz 2 In einer $\rho$-isometrischen Konfiguration mit dem gemeinsamen Inkreisradius $\rho^{*}$ gilt

$$
\frac{\rho^{*}}{\rho}=1-\frac{s_{E}}{s} .
$$




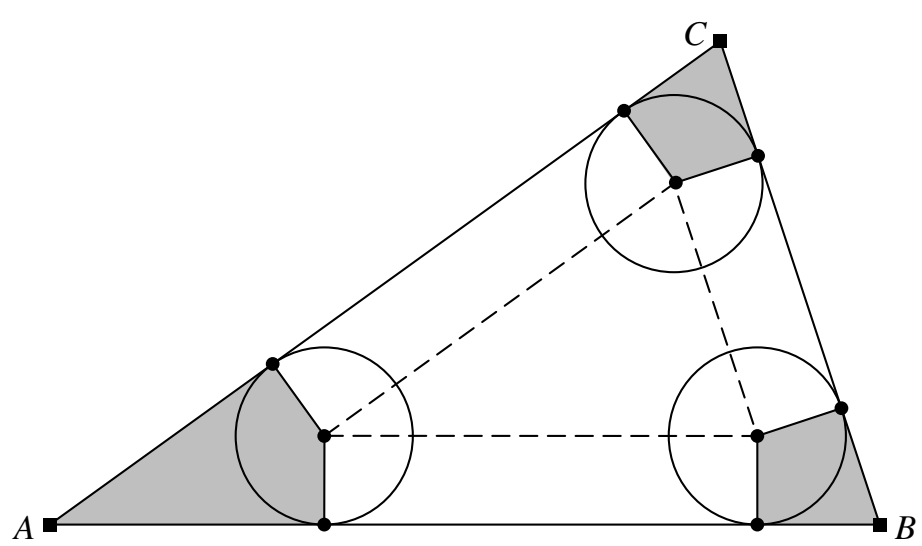

Fig. 11

Beweis. In Fig. 11 erkennt man leicht, dass sich bei gleichen Inkreisradien in den Ecken $A, B$ und $C$ die schraffierten Vierecke passend zu einem Dreieck $\Delta^{*}$ zusammen schieben lassen. Dieses Dreieck hat dann den Inkreisradius $\rho^{*}$ und ist ähnlich zum Ausgangsdreieck $\Delta$. Wegen der Ähnlichkeit gilt dann die Verhältnisgleichung

$$
\frac{\rho^{*}}{\rho}=\frac{U^{*}}{U}
$$

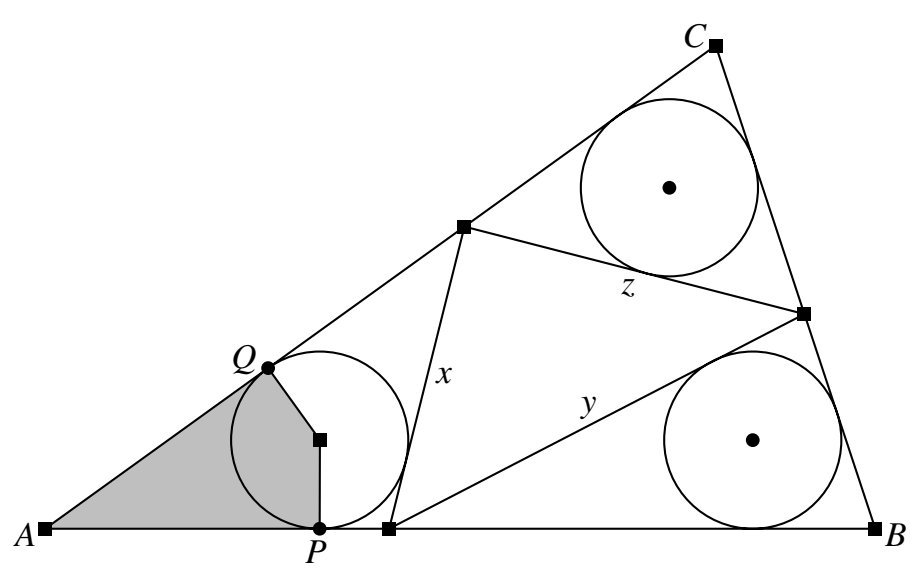

Fig. 12

Der Umfang $U^{*}$ setzt sich aber aus den Teilstücken der Seiten $a, b$ und $c$ zusammen, die von den Berührpunkten der Inkreise der Eckdreiecke begrenzt werden. Im Eckdreieck $\Delta_{A}$ (Fig. 12) gilt aber bekanntlich für die Strecken $A P$ und $A Q$

$$
A P=A Q=s_{A}-x \Longrightarrow A P+A Q=2 s_{A}-2 x .
$$


Addiert man diese Summe für alle drei Eckdreiecke, so erhält man den Umfang von $\Delta^{*}$ :

$$
U^{*}=2 s_{A}+2 s_{B}+2 s_{C}-2 x-2 y-2 z=U_{A}+U_{B}+U_{C}-2 U_{E} .
$$

Mit der allgemeinen Summenformel für die Umfänge der Teildreiecke ergibt sich daraus

$$
U^{*}=U-U_{E} .
$$

Setzt man dies in die Gleichung (7) ein, so erhält man die Behauptung.

Multipliziert man die beiden Formeln der Hilfssätze 1 und 2 beidseitig, so erhält man

$$
1=\left(1+\frac{2 s_{E}}{s}\right) \cdot\left(1-\frac{s_{E}}{s}\right) .
$$

Rechnet man die Klammern aus, so ergibt sich leicht die Gleichung

$$
s_{E}=\frac{1}{2} s .
$$

Setzt man dieses Ergebnis in die Gleichung des Hilfssatzes 2 ein, so folgt

$$
\rho^{*}=\frac{1}{2} \rho \text {. }
$$

Wir erhalten also

Hilfssatz 3 In einer $\rho$-isometrischen Konfiguration mit dem gemeinsamen Inkreisradius $\rho^{*}$ gilt

$$
s_{E}=\frac{1}{2} s \quad \text { und } \quad \rho^{*}=\frac{1}{2} \rho .
$$

Damit ist der erste Beweisschritt beendet. Wir können nun den folgenden Satz beweisen:

Satz 5 Es gibt keine nicht-triviale $\rho$-isometrische Konfiguration, also auch keine $\rho$ minimale-Konfiguration.

Beweis. Wie oben angekündigt, werden wir im zweiten Beweisschritt die Formel $F=\rho \cdot s$ verwenden, die wir nach $\rho$ auflösen. Mit Hilfssatz 3 ergibt sich dann für das Eckdreieck $\Delta_{A}:$

$$
\rho_{A}=\frac{1}{2} \rho \Longleftrightarrow \frac{F_{A}}{s_{A}}=\frac{1}{2} \cdot \frac{F}{s} \Longleftrightarrow 4 F_{A}=\frac{2 s_{A}}{s} \cdot F .
$$

Nun kommen wieder die Sterngrößen und die übliche Flächenformel zum Zuge:

$4 \cdot\left(\frac{1}{2}-b^{*}\right) \cdot b \cdot\left(\frac{1}{2}+c^{*}\right) \cdot c \cdot \sin \alpha=\frac{2 s_{A}}{s} \cdot b \cdot c \cdot \sin \alpha \Longleftrightarrow\left(1-2 b^{*}\right) \cdot\left(1+2 c^{*}\right)=\frac{2 s_{A}}{s}$.

Die Auflösung der Klammern führt zu

$$
\Longleftrightarrow-b^{*}+c^{*}=2 b^{*} c^{*}+\frac{s_{A}}{s}-\frac{1}{2} \quad\left(\Delta_{A}\right) .
$$


Nun wieder die zyklisch gebildeten Ergänzungen für die anderen Eckdreiecke:

$$
\begin{array}{ll}
\Longleftrightarrow-c^{*}+a^{*}=2 c^{*} a^{*}+\frac{s_{B}}{s}-\frac{1}{2} & \left(\Delta_{B}\right), \\
\Longleftrightarrow-a^{*}+b^{*}=2 a^{*} b^{*}+\frac{s_{C}}{s}-\frac{1}{2} & \left(\Delta_{C}\right) .
\end{array}
$$

Die Addition im fünften Beweisschritt liefert

$$
0=2 \cdot\left(b^{*} c^{*}+c^{*} a^{*}+a^{*} b^{*}\right)+\frac{s_{A}+s_{B}+s_{C}}{s}-\frac{3}{2} .
$$

Für den Restausdruck in dieser Gleichung gilt aber

$$
\frac{s_{A}+s_{B}+s_{C}}{s}-\frac{3}{2}=\frac{U_{A}+U_{B}+U_{C}-3 s}{U}=\frac{U+U_{E}-3 s}{U}=\frac{U_{E}-s}{U}=\frac{2 s_{E}-s}{U} .
$$

Der Zähler des letzten Bruchs ist aber nach dem Hilfssatz 3 gleich 0, so dass wir die Gleichung

$$
0=2 \cdot\left(b^{*} c^{*}+c^{*} a^{*}+a^{*} b^{*}\right)
$$

erhalten. Diese liefert wieder den üblichen Widerspruch, da alle Sterngrößen positiv sind.

\section{Ausblick}

Damit ist also gezeigt, dass es für die metrischen Dreiecksgrößen $F, U, \rho$ keine minimalen Konfigurationen geben kann. Als wesentliches Hilfsmittel für die Beweise diente der Hauptsatz über die minimalen Konfigurationen. Die strukturelle Einheitlichkeit der mit dem Hauptsatz durchgeführten Beweise lässt erwarten, dass er auch für andere metrische Dreiecksgrößen, die hier nicht untersucht wurden, hilfreich sein kann. Zu denken wäre an Größen wie

$$
a \cdot b \cdot c \text { oder } \rho_{a} \cdot \rho_{b} \cdot \rho_{c} \quad \text { oder } \rho_{a}+\rho_{b}+\rho_{c},
$$

wobei mit $\rho_{a}$ einer der drei Ankreisradien gemeint ist. Diese symmetrischen Terme treten bekanntlich in verschiedenen Formeln zur Dreiecksberechnung auf. Entscheidend für die Anwendung des Hauptsatzes ist aber immer die Frage, ob diese Größen monoton sind. Bei $a b c$ ist dies beispielsweise nicht der Fall, und wie schon bei der nicht-monotonen Dreiecksgröße $r$ lassen sich auch hier durchaus $a b c$-minimale Konfigurationen erzeugen. Ob hier ein allgemeinerer Zusammenhang zwischen der Monotonie der Messgröße und der Existenz minimaler Konfigurationen besteht, ist mir bisher nicht bekannt. Zu denken wäre aber an eine Vermutung der Art:

Vermutung Genau dann, wenn die metrische Dreiecksgröße $\mu$ als Funktion der Strecke $A^{\prime} C$ in Fig. 3 ein lokales Minimum hat, existiert eine $\mu$-minimale Konfiguration.

Dem interessierten Leser bleibt also noch ein weites Feld zur Erforschung konkreter metrischer Dreiecksgrößen, um zu einer möglichen allgemeinen Aussage zu gelangen. Über entsprechende Mitteilungen würde ich mich freuen. 


\section{Literatur}

[1] Bapist, P.: Die Entwicklung der neueren Dreiecksgeometrie. Mannheim 1992.

[2] Donath, E.: Die merkwürdigen Punkte und Linien des ebenen Dreiecks. Berlin 1968.

[3] Kazarinoff, N.D.: Analytic Inequalities. New York 1961 (Nachdruck 2003).

[4] Ogilvy, C.St.: Mathematische Leckerbissen. Über 150 noch ungelöste Probleme. Braunschweig 1969 (Originalausgabe: Tomorrows Math. New York 1962).

[5] Wittmann, E.Ch.: Elementargeometrie und Wirklichkeit. Braunschweig 1987.

Christian Schütz

Sandberg 47

D-47809 Krefeld, Deutschland

e-mail: sandberg47@t-online.de 